\title{
Una posible nueva clase trabajadora de servicios: evidencias a partir de un análisis del mercado de trabajo español entre 1999 y 2008
}

\author{
Juan Miguel GARCÍA NOGUEROLES \\ Universitat Autònoma de Barcelona \\ Departamento de Sociología \\ juanmiguel.garcia@uab.cat
}

Recibido: 12-03-2013

Aceptado: 10-02-2014

\begin{abstract}
Resumen: La clase trabajadora industrial ya no puede ser considerada como la hegemónica. Existe una nueva clase trabajadora de servicios que se ocupa principalmente en aquellos sectores intensivos en mano de obra, principalmente en el sector servicios de las economías desarrolladas. Sus características difieren de la clase obrera industrial: son mujeres, jóvenes, inmigrantes y sufren condiciones de trabajo que tienen que ver con la flexibilidad y la inestabilidad. Este artículo trata de mostrar para España la existencia de esta nueva clase obrera a partir de un doble análisis estadístico de la ECVT 1999 y 2008, utilizando las técnicas CHAID y Two-step cluter.
\end{abstract}

Palabras clave: clase trabajadora, precariedad, segmentación, servicios.

\section{A possible new working-class of services: evidences from a Spanish labour market analysis}

\begin{abstract}
Industrial working-class can't nowadays be considered as hegemonic. There is a new working-class of services that works in those sectors that use the manpower in an intensive way in developed societies. Its characteristics are different from the old working-class: they are women, young workers, immigrants and they are suffering working conditions that are related to flexibility and instability. This paper wants to show in Spain the existence of this new working-class with a statistical analysis of ECVT 1999 and 2008, using CHAID and two-step clustering.
\end{abstract}

Keywords: working-class, precariousness, segmentation, service sector.

\section{REFERENCIA NORMALIZADA}

García Nogueroles, J. M. (2014) "Una posible nueva clase trabajadora de servicios: evidencias a partir de un análisis del mercado de trabajo español entre 1999 y 2008". Cuadernos de Relaciones Laborales, Vol. 32, núm. 2, p. 413-437.

SUMARIO: Introducción. 1. Análisis de los datos. 1.1. Análisis clúster. 1.1.1. Descripción general de los conglomerados. 1.2. Análisis CHAID. 1.2.1. Sector productivo. 1.2.2. Tipo de contrato. 1.2.3. Salarios. 2. Conclusiones. 3. Bibliografía. 


\section{Introducción}

La propuesta de investigación que quiero plantear en este artículo se basa en la consideración de que podrían existir, al menos para el caso de España aunque probablemente también para otras sociedades económicamente avanzadas, unas nuevas clases trabajadoras de servicios y, dentro de ellas, un importante sector de características subproletarias que comparte condiciones de trabajo precarias y que se explica, en parte, por un, modo de acumulación basado en los bajos salarios de trabajadores con escasa capacidad de negociación de sus condiciones de trabajo. Estos colectivos de trabajadores, desde mi punto de vista, no solo no disminuyen su peso sino que lo están aumentando en los últimos años y son, además, básicos en la estructura productiva dado que desarrollan las tareas auxiliares que permiten el funcionamiento del resto de sectores. Formulo esta hipótesis inicial a partir de la constatación de dos fenómenos que vienen afectando con más intensidad a los mercados de trabajo de las sociedades avanzadas en las cuatro últimas décadas, aunque la lógica de funcionamiento de éstos encuentre sus raíces desde el mismo momento en que se generaliza el trabajo asalariado: la precarización de las condiciones de trabajo y la segmentación de los mercados de trabajo. Es por lo tanto de segmentación de los mercados de trabajo y de estratificación social a partir de la posición que ocupan los trabajadores en estos mercados de lo que va a hablarse en las próximas páginas. Se deja para futuros trabajos el establecimiento de las condiciones que estos trabajadores deben cumplir para ser conceptualizados como clase, aunque se propondrá en las conclusiones un primer marco analítico que trate de avanzar en este sentido.

La precarización laboral ha sido descrita por Cano $(1997,1998,2004)$ como un proceso multidimensional y complejo que implica un importante descenso en la calidad de vida del individuo que la sufre (Blanch, 2003) y que se intensifica a partir de la desregulación de los mercados de trabajo (Cano 2007, Cardenal 2006), implicando la generalización de las formas atípicas de empleo (Marshall, 1992) como el trabajo temporal (Recio, 2007) o a jornada parcial; fenómenos como el subempleo (OIT, 1998) o un empeoramiento de las condiciones materiales del trabajo (Pinilla 2004, Paoli 1999) que tiene también efectos sobre la salud (Amable, Benach, González, 2001) (Amable, Benach, 2007). Esta situación de precarización tiende a perpetuarse en el tiempo aunque los trabajadores pueden valorar que se trata de algo temporal (López Peláez, 2005). Los trabajadores que se sitúan en el ámbito de la precariedad ven afectados sus ingresos hasta el punto de poder situarse en situaciones cercanas a la pobreza -son los llamados working poor (Medialdea, Álvarez, 2005)- y suelen pertenecer a colectivos específicos -el de las mujeres es el ejemplo paradigmático, aunque hay que considerar también a jóvenes, inmigrantes, personas sin formación y otros- que desarrollan su actividad laboral en subsectores productivos muy concretos -algunas industrias, servicios como el comercio y la hostelería- (Banyuls et al, 2010). En este sentido la flexibilidad como estrategia empresarial y como ideología (Pollert, 1991) que se demanda en las relaciones laborales actuales afecta de manera especial a estos trabajadores precarios 
(Miguélez 2004 y 2005), de forma que es sobre ellos sobre los que se efectúan de forma más drástica los ajustes en cuanto a las necesidades de empleo de cada momento o en cuanto a la rentabilidad que las actividades económicas producen.

La segmentación de los mercados de trabajo es otro proceso complejo (Rubery, Wilkinson, 1981) (Rubery, 1978) (Dickens, Lang, 1992) producto de la misma tendencia, intrínseca al capital, para reclutar a sus trabajadores en mercados de trabajo cada vez más compartimentados, tanto a nivel interno -en una misma empresa, (Doeringer, Piore, 1985) (Burawoy, 1979)- como a nivel del mercado de trabajo general (Gordon, Edwards, Reich, 1986). Según esta teoría unos mercados de trabajo estarían compuestos por ocupaciones de mucha calidad, otros por ocupaciones de cariz más precario y otros combinarían características de los anteriores: estos mercados presentarían diferencias importantes entre ellos a nivel de retribuciones salariales, de estabilidad en la ocupación, de calificación requerida, etc. Desde mi punto de vista el hecho de la segmentación -más bien multisegmentación- es de vital importancia a la hora de entender el desarrollo de los colectivos precarios y el posible surgimiento de unas nuevas clases trabajadoras, pues su aislamiento de otras colectividades de trabajadores será un elemento básico por entender su debilidad y su posición en el mercado de trabajo. En todo caso hay que estudiar atentamente estas diferentes condiciones de trabajo que tienen unos $\mathrm{u}$ otros trabajadores (Alós, 2007) ya que la constatación de estas diferencias no permite que las propias diferencias sean consideradas de forma automática como muestra de la segmentación: solo las diferencias que provienen de estrategias empresariales, de elementos relacionados con la oferta de trabajo o incluso de aspectos institucionales pueden ser consideradas evidencia de la segmentación.

En conclusión, la segmentación de los mercados de trabajo es un proceso muy importante por varias razones: la primera porque, como ya se ha comentado, es una estrategia del capital dirigida a la debilitación de las capacidades de negociación de los trabajadores (Kern, Schumann, 1988). Efectivamente, lo que está sucediendo es que la negociación colectiva -entre capital y trabajo- y los pactos a tres bandas entre gobierno, patronal y sindicatos son cada vez menos necesarios: ahora las negociaciones tienden a hacerse de manera individual con el trabajador, si estamos hablando de los segmentos primarios independientes, o no hay ningún tipo de negociación-simplemente se hace referencia al ejército de reserva que codicia esas ocupaciones, la ley de la oferta y la demanda- en las ocupaciones de los secundarios, donde los convenios colectivos sectoriales o provinciales van perdiendo peso a favor de los de empresa (Recio, 2011).

La segunda razón de la importancia de este proceso vendría relacionada con la existencia de distintos vectores de discriminación en el mercado de trabajo: de esta manera las mujeres o los inmigrantes (Iglesias, Llorente, 2006) se encuentran, entre otros colectivos, sobrerepresentados en ocupaciones de los segmentos secundarios. La tercera razón podríamos relacionarla con una hipotética tendencia a la desaparición del segmento primario subordinado -el primario dependiente de Gordon, Edawars y Reich (1986)- y al crecimiento de los segmentos secundarios y precarios (Braverman, 1974), (Baglioni, 1992). Siguiendo la anterior idea, un 
análisis exhaustivo lo que muestra es la multitud de mundos laborales -no solo en cuanto a segmentos secundarios (Alós, Jodar, 2005)- que podríamos describir con el término multisegmentación. La cuarta y última razón de la importancia de este proceso de segmentación podemos explicarla por la permanencia en la precariedad de grupos sociales cada vez más amplios y durante períodos cada vez mayores de tiempo.

\section{Análisis de los datos}

Es de suponer que un análisis del mercado de trabajo español, desde la perspectiva de la segmentación, hará visibles los principales grupos de trabajadores según sus condiciones de empleo, así como ofrecerá una lógica interna que justificará esta división. Este análisis se ha hecho a partir de los datos de la ECVT de 1999 y de 2008, con el objetivo de ofrecer una comparación entre dos momentos especialmente significativos en la evolución del mercado de trabajo español, ya que representan el punto de inicio y de final de un periodo expansivo para la economía española, dónde quizás cabría suponer una mejora de las condiciones de trabajo para todos los trabajadores aunque esto, a la vista de los datos, podría no estar sucediendo de esa manera.

El análisis se ha hecho a partir de una doble técnica: el análisis de conglomerados en dos etapas (two step cluster) que nos permite definir diferentes grupos de sujetos de acuerdo a las semejanzas y diferencias en la medición de las variables que se introducen en el análisis, por un lado, y el CHAID (Chi Square Automatic Interaction Detection) que jerarquiza las variables independientes que actúan en la determinación de otra variable considerada como dependiente, por el otro lado.

Se han considerado las siguientes variables para el análisis de conglomerados. Hay que tener en cuenta que las variables se han seleccionado para cubrir diferentes aspectos de la medición de las condiciones de trabajo objetivas (variables contractuales, organizativas o sindicales) o subjetivas (variables de satisfacción). Además se incluye un grupo de variables estructurales que ayudan a definir al trabajador en clave de colectivo (edad, sexo, estudios, país de nacimiento) o a la empresa en la que éste está ocupado (sector productivo, titularidad de la empresa, tamaño de la empresa). 


\begin{tabular}{|l|l|l|l|}
\hline Tipo & Variable & $\begin{array}{l}\text { ECVT } \\
\mathbf{1 9 9 9}\end{array}$ & $\begin{array}{l}\text { ECVT } \\
\mathbf{2 0 0 8}\end{array}$ \\
\hline Estructural & Edad & V14 & V5 \\
\hline Estructural & Tamaño empresa & V21 & V12b \\
\hline Estructural & Sector productivo & V18 & V9 \\
\hline Estructural & $\begin{array}{l}\text { Titularidad de la empresa: } \\
\text { pública/privada }\end{array}$ & V19 & V10 \\
\hline Estructural & Formación & V257 & V7 \\
\hline Estructural & Género & V253 & V6 \\
\hline Estructural & País de nacimiento & V67b & V23 \\
\hline Contractual & $\begin{array}{l}\text { Tipo de contrato (temporal o } \\
\text { indefinido) }\end{array}$ & V24 & V14 \\
\hline Contractual & Salario & V194 & V71 \\
\hline Satisfacción & Satisfacción general & V92 & V27 \\
\hline Satisfacción & Satisfacción salarial & V189 & V28a \\
\hline Satisfacción & $\begin{array}{l}\text { Satisfacción con } \\
\text { organización del trabajo }\end{array}$ & V109 & V28m \\
\hline Organizativa & Horas trabajadas & V150 & V59 \\
\hline Organizativa & $\begin{array}{l}\text { Posición en la estructura de } \\
\text { la empresa }\end{array}$ & V23 & V11 \\
\hline Organizativa & $\begin{array}{l}\text { Tipo de jornada (partida } \\
\text { continua) }\end{array}$ & V145 & V55 \\
\hline Sindical & $\begin{array}{l}\text { Afiliación a sindicatos } \\
\text { V216, }\end{array}$ & V47 \\
\hline & V217, & \\
\hline
\end{tabular}

Los análisis CHAID refinan los resultados ofrecidos por el clúster y explican algunas relaciones que se dan entre las variables identificadas, dado que la técnica CHAID permite establecer una de las variables como dependiente. En este sentido las variables que se han definido como dependientes han sido el sector productivo, los salarios y el tipo de contrato (temporal o indefinido). El paquete de variables independientes ha estado formado por la edad, el tamaño de la empresa, formación, género, país de nacimiento y el sector productivo. Cuando el sector productivo ha funcionado como variable dependiente no lo ha hecho, evidentemente, dentro del paquete de las independientes. 


\subsection{Análisis clúster}

En primer lugar es importante señalar que existen otras investigaciones que han realizado procedimientos de análisis similares y que han obtenido resultados que considero que van en la línea de los que presento en este trabajo: Alòs, en su tesis doctoral (Alòs, 2007), distingue para el caso español y con datos de 2004 hasta 6 segmentos. Según López Roldán (López Roldán, 1996), con datos de inicios de la década de los noventa y solo para la región metropolitana de Barcelona, se distinguen cuatro segmentos a los que se debería añadir un quinto segmento marginal. El más reciente (Prieto, 2009) define también para el mercado de trabajo español tres segmentos aunque partiendo de una subdivisión inicial en seis, de los cuales tres de ellos se corresponderían con el segmento secundario del mercado de trabajo tal y como ha sido definido por Gordon Edwards y Reich (1986).

De acuerdo con el análisis que realizado se han identificado seis clústeres o conglomerados de trabajadores, con algunas variaciones entre 1999 y 2008 -en cuanto al tamaño de los conglomerados, pero también en cuanto a su composiciónque justifican desde mi punto de vista el que se pueda hablar de la consolidación de una clase precarizada de trabajadores de servicios. Una primera mirada a la distribución por conglomerados (tabla 1) muestra que ha habido un incremento entre aquellos conglomerados a que se disponen en la parte más alta de la distribución, lo que vendría a significar que durante el transcurso del período estudiado las condiciones de trabajo pueden haber mejorado. En todo caso, y considerando las distribuciones por tercios, en 1999 el tercio con mejores condiciones de trabajo agrupaba a un $26{ }^{\prime} 5 \%$ de los trabajadores frente a un $31^{\prime} 4 \%$ que agrupaba en 2008. El segundo tercio de 1999 agrupaba a un $32 \%$ y en 2008 agrupaba un $33,4 \%$ de los trabajadores. Finalmente el tercio con peores condiciones de trabajo suponía en 1999 un 41'5\% y en 2008 un 35'2\% de los trabajadores. Hay que tener en cuenta, sin embargo, que el peor conglomerado, el sexto, el que he nombrado como segmento secundario débil, se ha mantenido estable en los dos momentos estudiados -un $21^{\prime} 3 \%$ en 1999 y un $22{ }^{\prime} 5 \%$ en 2008 - y que en ambos momentos es el conglomerado que agrupa a un mayor número de trabajadores, especialmente en 2008 donde las diferencias con los otros conglomerados son mayores. Esta sería la primera evidencia de que el proceso de expansión económica entre 1999 y 2008 no ha supuesto los mismos avances para todos los trabajadores.

Tabla 1. Distribución de los trabajadores entrevistados en conglomerados para 1999 y y 2008.

\begin{tabular}{|l|l|l|}
\hline Conglomerados/Segmentos & $\mathbf{1 9 9 9}$ & $\mathbf{2 0 0 8}$ \\
\hline Primario Independiente Superior & $11,2 \%$ & $12,9 \%$ \\
\hline Primario Independiente Inferior & $15,3 \%$ & $18,5 \%$ \\
\hline Primario Dependiente de Servicios & $12,4 \%$ & $16,3 \%$ \\
\hline Primario Dependiente Industrial & $19,6 \%$ & $17,1 \%$ \\
\hline Secundario de transición & $20,2 \%$ & $12,7 \%$ \\
\hline Secundario débil & $21,3 \%$ & $22,5 \%$ \\
\hline
\end{tabular}

Fuente: elaboración propia a partir de los datos de la ECVT 1999 y 2008 


\subsubsection{Descripción general de los conglomerados}

Una descripción general de los conglomerados obtenidos nos ofrece los siguientes resultados:

A) Primario independiente superior. Este segmento es el que presenta mejores condiciones de trabajo tanto para 1999 como para 2008. Este primer segmento, pero también el segundo, teniendo en cuenta que son segmentos primarios independientes, tienen ciertas características en cuanto al trabajo concreto autonomía en el trabajo, buenas retribuciones, necesidad de formaciones o calificaciones (formales o no) elevadas que hacen a estos trabajadores difícilmente intercambiables- que se relacionan con altas productividades y situaciones de privilegio en el mercado de trabajo. Los trabajadores son de edades maduras, con especial peso para aquellas edades que se sitúan alrededor de los 45 años. Mayoritariamente son españoles, ocupados en empresas grandes y con estudios universitarios. Gozan de contratos indefinidos y de altos salarios. Generalmente se muestran satisfechos con las condiciones de su puesto de trabajo y se ocupan en los servicios.

Existen, sin embargo, algunas diferencias entre 1999 y 2008. Quizás la principal es que en 1999 estamos hablando de un conglomerado eminentemente privado mientras que en 2008 lo público supone dos terceras partes del total de estos empleados. Esto creo que puede estar producido porque hay un desplazamiento de las mejores condiciones de trabajo desde el sector privado hasta el sector público, desplazamiento que podría no estar provocado por una importante mejora en las condiciones de trabajo de lo público sino por un cierto retroceso en las condiciones de trabajo del ámbito privado, a pesar de estar en un período expansivo en la economía española. Esta mayor presencia de trabajo público en 2008 es el motivo de que el porcentaje de trabajo de servicios sea más importante en 2008 que en 1999 -el trabajo de titularidad pública es en su gran mayoría de servicios- y esta mayor terciarización del mejor conglomerado es lo que produce que la proporción de mujeres sea mayor en 2008 que en 1999. En el mismo sentido también explica las mayores tasas de sindicación y el hecho de que los horarios mayoritarios para 2008 se extiendan menos que en 1999. En cuanto a las diferencias, para acabar, también hay que comentar que en 1999 respecto la posición en la estructura de la empresa se encuentran muchos más directivos, supervisores y encargados que en 2008. Una explicación a esto podría estar relacionada con la titularidad de la empresa: podría haber un mayor número de directivos y supervisores entre los trabajadores del ámbito privado situados en el segmento primario independiente que entre los trabajadores públicos situados en este mismo segmento

B) Primario independiente inferior. Es un conglomerado de trabajadores donde la distribución de la edad tiene porcentajes superiores de trabajadores más jóvenes que en el anterior segmento, de hecho éstos se sitúan mayoritariamente entre los 30 y los 40 años. Los salarios se sitúan principalmente en las categorías más altas si bien en 1999 hay un importante porcentaje de salarios medios. Son principalmente 
nacidos en España y están ocupados en empresas grandes -más de 250 trabajadores-, con formación universitaria (especialmente en 1999) y con contratos indefinidos, si bien en el conglomerado de 1999 la tasa de temporalidad era bastante importante dado que superaba el $20 \%$. El grado de afiliación sindical es elevado en ambos momentos y los trabajadores se muestran satisfechos con sus condiciones de trabajo. Por lo que respecta a las diferencias hay que tener en cuenta lo mismo que en el conglomerado anterior: en este caso para 1999 nos encontramos con un mayor porcentaje de trabajo público que a su vez hace que en 1999 haya más trabajo de servicios y más mujeres así como que las horas trabajadas sean, en general, menos. Si para 1999 este segundo escalón en cuanto a la calidad del trabajo tiene un importante peso de lo público y para 2008 el peso es mayoritario por lo que respecta a lo privado la explicación puede ir en la línea de lo que hemos ensayado anteriormente, es decir: las condiciones de trabajo para lo público en 1999 no superaban en muchos de los casos a las que, en los empleos de mayor calidad, se podían encontrar en los sectores de titularidad privada; esto parece haber desaparecido para 2008, probablemente por un cierto retroceso del sector privado hacia el público, por lo menos en lo que respecta a las condiciones de trabajo.

En la misma dirección que en el anterior conglomerado pero en sentido contrario, hay que señalar que para 2008 muchos de los trabajadores tienen posiciones de dirección o supervisión, hecho que no se detecta con la misma intensidad para 1999. Esto es importante en tanto en cuanto se observa -también en el anterior clústercomo las mejores condiciones de trabajo en el sector privado se relacionan de forma directa con el hecho de dirigir equipos o de tener un lugar de responsabilidad, cosa que no sucede con la misma intensidad en el ámbito público.

C) Primario dependiente de servicios. En este tercer conglomerado las ocupaciones tienen menores requerimientos de formación y los trabajadores que las desarrollan podrían ser más fácilmente intercambiables ya que existe la posibilidad de que no estén tan especializados como los trabajadores de los segmentos independientes (en todo caso tendríamos que considerar la existencia de actividades formativas informales y/o de la formación dentro de la empresa para ver si este análisis se confirma); de todas formas estamos aún hablante de ocupaciones bastante estables y con ciertas características que pueden entenderse como de calidad en el empleo. Los trabajadores que se clasifican dentro este clúster son trabajadores que se sitúan de forma mayoritaria entre los 40 y los 50 años de edad, nacidos en España, que trabajan principalmente a los servicios, en empresas de más de 250 trabajadores y principalmente de titularidad pública (especialmente para 2008). Son trabajadores que perciben un salario conceptualizado entre los medios y hay que señalar que son los trabajadores que más se sindican, superando grados de afiliación del $40 \%$. Son también trabajadores que gozan de contratos indefinidos en una muy alta proporción, si bien la temporalidad en 2008 roza el $20 \%$. En cuanto a las diferencias entre 1999 y 2008 hay que señalar que para 2008 hay más porcentaje de mujeres que de hombres, hecho que creo que debe ver con el mayor peso del ámbito público en 2008. De la misma manera en 1999 los trabajadores de este 
segmento alargan más los horarios y para 2008 la formación es sensiblemente más elevada que en 1999. En este sentido hay que señalar que lo que podría estar pasando es que en 1999 aún se encuentran importantes colectivos de trabajadores dentro de los segmentos de calidad en el trabajo con formaciones bajas y que responden de manera más clara al perfil primario dependiente según había estado descrito en las teorías de la segmentación, mientras que para 2008 este escalón de calidad en el empleo ya estaría adscrito con más intensidad a trabajadores con formaciones superiores, con mayor peso de servicios y del ámbito público, lo que puede sugerir una desintegración de los segmentos primarios dependientes tradicionales. Por lo que respecta a la satisfacción, los trabajadores de este segmento se encuentran más satisfechos en 1999 que en 2008, hecho que creo que debe ver con que las expectativas generadas después de los años de crecimiento económico no han acabado de consolidarse en las condiciones de trabajo que los ocupados consideraban adecuadas.

D) Primario dependiente industrial. Este conglomerado incluye a los trabajadores de la construcción que a lo largo del período experimentarán un incremento importante aunque la destrucción de empleo, como ya sabemos, se centró muy especialmente en ellos al inicio de la crisis. Es un conglomerado que concentra a trabajadores masculinos, ocupados en empresas de menos de 50 trabajadores (en muchos casos de menos de 10 trabajadores) y de titularidad privada. El peso de las ocupaciones de servicios es muy bajo, especialmente si lo comparamos con el peso real de los servicios en la estructura productiva. Disponen de salarios que están conceptualizados entre los medios y su tasa de temporalidad se encuentra alrededor del $25 \%$ de los efectivos, lo que ya supone una tasa muy próxima a la media establecida para cada año en la ECVT. Son trabajadores con formaciones modestas -entre primaria y secundaria- que se muestran satisfechos con sus condiciones de trabajo y con la organización de este trabajo. En cuanto a los horarios de trabajo, éste ocupa mayoritariamente entre 40 y 49 horas a la semana, pero es importante señalar que hay un importante porcentaje de estos trabajadores que alarga su horario por encima de las 50 horas, lo que podría explicar que en muchos casos los salarios sean salarios medios aún siendo ocupaciones para las que no hace falta una formación específica. Por lo que respecta a las diferencias que podemos encontrar entre 1999 y 2008 creo que las más importantes hacen referencia a la edad y a la procedencia de los trabajadores: por un lado para 1999 la distribución de las edades se concentra en edades un poco más jóvenes que en 2008; por otro lado la presencia de trabajadores extranjeros, que en 1999 es insignificante, toma para 2008 una gran importancia, lo que es muestra de la llegada y la consolidación de este contingente de trabajadores. Otra de las diferencias es que con los salarios los trabajadores del primario dependiente industrial de 2008 se muestran menos satisfechos que los de 1999 y que se sindican en una proporción muy superior; de tal forma que se podría suponer que el período de expansión económica ha hecho que las expectativas de estos trabajadores respecto algunas condiciones de 
trabajo hayan crecido y que, al no verlas completamente satisfechas, algunos hayan podido tomar la decisión de sindicarse para mejorarlas.

E) Secundario de transición. Es posiblemente el segmento que presenta mayores diferencias entre 1999 y 2008; hecho de una gran importancia para la interpretación de los datos que ensayo en este artículo. El nombre de transición debe ver no únicamente con que se trata de un clúster donde las malas condiciones de trabajo ya se conforman como próximas a las de un segmento secundario tal y como estuvo descrito en las teorías de la segmentación (Gordon, Edwards, Reich, 1986) o a las de un segmento secundario débil, por utilizar terminología de otros autores (Alós, 2007), sino también porque en él se muestra el paso de unas categorías de trabajadores que están en situaciones precarias o muy próximas a la precariedad, a otras categorías de trabajadores que, sin formar parte de los mismos colectivos, se encuentran en situaciones análogas de precariedad: las que aunque no han llegado al extremo de total precariedad dentro del mercado de trabajo, se encuentran muy próximas a ella.

En este sentido nos encontramos delante de trabajadores ocupados en empresas pequeñas, de titularidad privada, que reciben salarios medios o bajos y con tasas de temporalidad bastante elevadas, especialmente las de 1999 que superan el 35\%. Una gran parte de estos trabajadores tienen formaciones muy modestas, pero creo que es importante señalar que un $20 \%$ de los trabajadores clasificados en el segmento secundario de transición de 2008 están formados a nivel universitario o tienen una calificación superior de secundaria, lo que muestra de alguna manera que las elevadas formaciones cada vez son menos decisivas a la hora de asegurar una carrera laboral de éxito. Ya hay una presencia significativa de trabajadores extranjeros en 1999, presencia que aumenta para 2008; y las características masivamente industriales o de construcción de 1999 dejan paso a una terciarización muy importante del conglomerado en 2008, con una importante presencia de los servicios manuales que ya estaban presentes en 1999. Esta terciarización también provoca que el secundario de transición pase de ser un conglomerado eminentemente masculino en 1999 a un conglomerado donde hombres y mujeres están presentes en porcentajes mucho más equilibrados. Esta mayor presencia de mujeres; la mayor presencia de actividades productivas de servicios; la distribución por edades más equilibrada en 2008 que en 1999 aunque siempre con tendencia hacia trabajadores por debajo de los 45 años; la mayor presencia de extranjeros en 2008; la existencia de un $20 \%$ de los trabajadores del clúster que para 2008 se ocupaban en empresas de más de 250 trabajadores frente a los menos de un $10 \%$ en 1999 y una mucho más importante presencia de trabajadores que se ocupan en jornadas parciales (aunque lo normal en 1999 y en 2008 es alargar la jornada por encima de las 40 horas) también para 2008 creo que es muestra de una importante heterogeneización de la clase trabajadora que se encuentra en las puertas de la precariedad o ya inmersa en ella.

Es necesario comentar que los grados de satisfacción de los trabajadores del secundario de transición de 2008 son mucho más elevados que la satisfacción 
expresada para 1999 y, paralelamente, la tasa de sindicación de 1999 casi triplica la de 2008. La explicación a esto puede ir en la línea de suponer que la llegada de muchos trabajadores de los grupos débiles en el mercado de trabajo, que ya se podrían mostrar satisfechos con el único objetivo de tener un empleo, sin preocuparse mucho por la calidad de éste, ha acabado reduciendo o diluyendo el descontento respeto las malas condiciones de trabajo. Estos trabajadores también pueden tener expectativas más bajas o pueden considerar sus puestos de trabajo como provisionales, de tal manera que no se implican en la defensa de las condiciones de éstos. Sea como sea lo cierto es que los empleos o las situaciones que dan lugar a formar parte del colectivo de trabajadores más próximo a la precariedad se ha diversificado de forma importante en el período estudiado. Es muy importante comentar que el segmento secundario de transición en 1999 tiene muchos elementos que lo acercan a la definición de un segmento primario dependiente, aunque más precario; sin embargo para 2008 el segmento secundario de transición es muy similar al segmento secundario débil y comparte con él las características propias de los segmentos secundarios definidas por los teóricos de la segmentación.

F) Secundario débil. El segmento secundario débil no ha cambiado tanto entre 1999 y 2008 como el segmento secundario de transición. En el extremo inferior de las condiciones de trabajo continuamos estando ante un colectivo eminentemente de trabajadoras jóvenes que están ocupadas en servicios y con una importante presencia de servicios manuales, en empresas de titularidad privada, en las que reciben bajos salarios y donde sufren una importante tasa de temporalidad así como de contratación parcial, aunque lo normal es que este clúster de trabajadores alargue su jornada semanal por encima de las 40 horas. Ya en 1999 había una presencia significativa de trabajadoras nacidas en el extranjero, pero esta presencia se dispara en 2008 hasta superar el $20 \%$ de los efectivos. Son trabajadores (trabajadoras muy frecuentemente) ocupados en empresas pequeñas, muy a menudo de menos de 10 trabajadores, aunque hay que señalar que para 2008 hay una presencia importante de los que se ocupan en empresas de más de 250 trabajadores; esto, que también sucedía en el anterior conglomerado, sugiere que la protección respeto las buenas condiciones de trabajo que ofrecían las empresas de tamaños importantes puede haber estado desapareciendo, por lo menos para determinados sectores o colectivos. Finalmente, hay un importante porcentaje de ocupados (alrededor de un tercio del total, tanto en 1999 como en 2008) que tienen estudios secundarios superiores o universitarios, lo que es muestra de que la formación, por lo menos en el caso de las mujeres, no ha servido en los últimos años para escapar de la precariedad.

Las diferencias entre 1999 y 2008 se relacionan principalmente con la satisfacción expresada y en el grado de sindicación; en este caso son los trabajadores de 2008 los que muestran mayores porcentajes de sindicación y de baja satisfacción. Este traslado del descontento, en 2008, desde el segmento secundario de transición hasta el segmento secundario débil puede ser la muestra de una extensión de la conciencia de la dificultad de escapar de la precariedad en ciertos 
colectivos - los más desfavorecidos- y, por tanto, de la necesidad de hacer algo, lo que explicaría la mayor tasa de afiliación sindical. Entre 1999 y 2008 hay algunas modificaciones en la composición de este conglomerado, pero creo que hay que tener en cuenta el anterior segmento -secundario de transición- para valorar adecuadamente el proceso de heterogeneización de los trabajadores del secundario, especialmente de aquellos que se dedican a actividades de servicios. Actualmente, así, nos encontramos ante unos conglomerados donde la presencia masculina ya no es tan extraña, donde hay efectivos de distintas nacionalidades, de distintas edades, con distintas formaciones y que viven su situación laboral de formas muy diferentes, lo que supone un elemento importante de complejización del análisis, por lo menos a nivel social; aunque la existencia de un mayor número de mujeres e inmigrantes en estos conglomerados es otro ejemplo de la etnosegmentación y de la segmentación por género que es característica del mercado de trabajo español en los últimos años.

Los trabajadores de los segmentos secundarios son trabajadores que, por lo que respecta a las condiciones contractuales, van a sufrir contratos temporales con mucha más frecuencia que otros trabajadores y, además, dispondrán de más bajos salarios. Tendrán también jornadas parciales en mucha más proporción. La evolución entre 1999 y 2008 muestra que en cuanto a la temporalidad la situación no ha empeorado -hay que recordar que durante esta década ha habido actuaciones de la administración en la dirección de fomentar la contratación indefinida, en un contexto de crecimiento económico- pero es necesario señalar como para 2008 aún hay altos grados de temporalidad en los peores clústeres. Por lo que respecta a los salarios la situación sí parece haber empeorado, por lo menos para este segmento secundario débil: los valores de bajos salarios afectan a casi el $75 \%$ de sus efectivos cuando al 1999 estos bajos salarios eran un 57'65\% del total del sexto clúster.

En cuanto a la satisfacción la principal diferencia a que podemos encontrar entre 1999 y 2008 es la aparición en 2008 de un perfil secundario débil con valores de insatisfacción para las distintas variables consideradas sensiblemente superiores a los otros clústeres y, especialmente, a los del perfil secundario de transición. La composición a nivel de variables de control muestra un importante peso de colectivos denominados débiles, pero un análisis del resto de las variables también muestra que las condiciones de trabajo, en general, son peores. La insatisfacción, así, viene o por las peores condiciones de trabajo, o por la existencia de colectivos diferentes que tienen aspiraciones y demandas diferentes y que están más predispuestos a expresar insatisfacción, o por una combinación de ambas causas; pero el caso es que sí parece evidente que aparece dentro de la nueva clase trabajadora, incluyendo los que trabajan en los servicios manuales tal y como se han descrito, un grupo que muestra su descontento con las condiciones de trabajo. En cualquier caso los resultados del análisis sí muestran grupos de trabajadores, con un peso determinante de los trabajadores en servicios, que presentan grados de satisfacción que en algunos casos -especialmente para 2008- están lejos de los grados de satisfacción del resto de los trabajadores; esto creo que es otro indicador 
de la existencia, de forma diferenciada, de la clase trabajadora de servicios que defiendo.

Tabla 2. Distribución de los trabajadores de acuerdo con la titularidad de la empresa en la que trabajan según conglomerado.

\begin{tabular}{|l|r|r|r|r|}
\hline \multicolumn{4}{|l|}{$\mathbf{1 9 9 9}$} & \multicolumn{2}{l|}{$\mathbf{2 0 0 8}$} \\
\hline & \multicolumn{1}{|l|}{$\begin{array}{l}\text { Titularidad } \\
\text { pública }\end{array}$} & $\begin{array}{l}\text { titularidad } \\
\text { privada }\end{array}$ & $\begin{array}{l}\text { Titularidad } \\
\text { pública }\end{array}$ & \multicolumn{1}{l|}{$\begin{array}{l}\text { titularidad } \\
\text { privada }\end{array}$} \\
\hline Primario Independiente Superior & $15,81 \%$ & $84,19 \%$ & $68,30 \%$ & $31,70 \%$ \\
\hline Primario Independiente Inferior & $83,84 \%$ & $16,16 \%$ & $1,35 \%$ & $98,65 \%$ \\
\hline Primario Dependiente de Servicios & $52,67 \%$ & $47,33 \%$ & $77,48 \%$ & $22,52 \%$ \\
\hline Primario Dependiente Industrial & $1,36 \%$ & $98,64 \%$ & $3,08 \%$ & $96,92 \%$ \\
\hline Secundario de transición & $2,41 \%$ & $97,59 \%$ & $12,00 \%$ & $88,00 \%$ \\
\hline Secundario débil & $4,16 \%$ & $95,84 \%$ & $3,64 \%$ & $96,36 \%$ \\
\hline
\end{tabular}

Fuente: elaboración propia a partir de los datos de la ECVT 1999 y 2008

Tabla 3. Distribución de los trabajadores de acuerdo con el sector económico de la empresa en la que trabajan según conglomerado.

\begin{tabular}{|l|l|l|l|l|l|l|l|}
\hline & \multicolumn{2}{|l|}{1999} & 2008 & \multicolumn{2}{|c|}{} \\
\hline & $\begin{array}{l}\text { Resto del } \\
\text { sistema } \\
\text { productivo } \\
\text { servicios } \\
\text { (Servicios } \\
\text { no } \\
\text { clasificados } \\
\text { como } \\
\text { manuales) }\end{array}$ & $\begin{array}{l}\text { Servicios } \\
\text { manuales }\end{array}$ & $\begin{array}{l}\text { NS/ } \\
\text { NC }\end{array}$ & $\begin{array}{l}\text { Resto del } \\
\text { sistema } \\
\text { productivo }\end{array}$ & $\begin{array}{l}\text { Resto } \\
\text { servicios } \\
\text { (Servicios } \\
\text { no } \\
\text { clasificados } \\
\text { como } \\
\text { manuales) }\end{array}$ & $\begin{array}{l}\text { Servicios } \\
\text { manuales }\end{array}$ \\
\hline $\begin{array}{l}\text { Primario Independien- } \\
\text { te Superior }\end{array}$ & $32,41 \%$ & $57,71 \%$ & $8,10 \%$ & $\begin{array}{l}1,78 \\
\%\end{array}$ & $2,39 \%$ & $97,49 \%$ & $0,11 \%$ \\
\hline $\begin{array}{l}\text { Primario Independien- } \\
\text { te Inferior }\end{array}$ & $0,29 \%$ & $98,99 \%$ & $0,14 \%$ & $\begin{array}{l}0,58 \\
\%\end{array}$ & $52,14 \%$ & $38,35 \%$ & $9,51 \%$ \\
\hline $\begin{array}{l}\text { Primario Dependiente } \\
\text { de Servicios }\end{array}$ & $29,18 \%$ & $65,30 \%$ & $2,49 \%$ & $\begin{array}{l}3,02 \\
\%\end{array}$ & $2,25 \%$ & $97,21 \%$ & $0,54 \%$ \\
\hline $\begin{array}{l}\text { Primario Dependiente } \\
\text { Industrial }\end{array}$ & $69,34 \%$ & $10,29 \%$ & $19,12 \%$ & $\begin{array}{l}1,24 \\
\%\end{array}$ & $80,92 \%$ & $10,95 \%$ & $8,13 \%$ \\
\hline $\begin{array}{l}\text { Secundario de transi- } \\
\text { ción }\end{array}$ & $65,32 \%$ & $19,47 \%$ & $14,33 \%$ & $\begin{array}{l}0,88 \\
\%\end{array}$ & $30,91 \%$ & $36,68 \%$ & $32,41 \%$ \\
\hline \begin{tabular}{l} 
Secundario débil \\
\hline
\end{tabular} & $15,40 \%$ & $34,24 \%$ & $48,60 \%$ & $\begin{array}{l}1,77 \\
\%\end{array}$ & $25,50 \%$ & $41,90 \%$ & $32,60 \%$ \\
\hline
\end{tabular}

Fuente: elaboración propia a partir de los datos de la ECVT 1999 y 2008 
Tabla 4. Distribución de los trabajadores de acuerdo con el tipo de contrato que ostentan según conglomerado.

\begin{tabular}{|l|l|l|l|l|l|}
\hline \multicolumn{7}{|l}{1999} & \multicolumn{2}{l|}{$\mathbf{2 0 0 8}$} \\
\hline & indefinido & temporal & NS/NC & indefinido & temporal \\
\hline Primario Independiente Superior & $93,08 \%$ & $3,16 \%$ & $3,75 \%$ & $93,16 \%$ & $6,84 \%$ \\
\hline Primario Independiente Inferior & $76,05 \%$ & $21,36 \%$ & $2,60 \%$ & $95,88 \%$ & $4,12 \%$ \\
\hline Primario Dependiente de Servicios & $94,66 \%$ & $4,09 \%$ & $1,25 \%$ & $80,09 \%$ & $19,91 \%$ \\
\hline Primario Dependiente Industrial & $71,15 \%$ & $23,30 \%$ & $5,54 \%$ & $75,53 \%$ & $24,47 \%$ \\
\hline Secundario de transición & $58,97 \%$ & $37,86 \%$ & $3,17 \%$ & $73,13 \%$ & $26,87 \%$ \\
\hline Secundario débil & $47,55 \%$ & $47,35 \%$ & $5,10 \%$ & $62,52 \%$ & $37,48 \%$ \\
\hline
\end{tabular}

Fuente: elaboración propia a partir de los datos de la ECVT 1999 y 2008

Tabla 5. Distribución de los trabajadores de acuerdo con el salario según conglomerado.

\begin{tabular}{|c|c|c|c|c|c|c|c|c|c|}
\hline & \multicolumn{5}{|l|}{1999} & \multicolumn{4}{|l|}{2008} \\
\hline & $\begin{array}{l}\text { Bajos } \\
\text { salarios }\end{array}$ & $\begin{array}{l}\text { Salarios } \\
\text { medios }\end{array}$ & $\begin{array}{l}\text { salarios } \\
\text { altos }\end{array}$ & $\begin{array}{l}\text { sala- } \\
\text { rios } \\
\text { muy } \\
\text { altos }\end{array}$ & NS/NC & $\begin{array}{l}\text { Bajos } \\
\text { salarios }\end{array}$ & $\begin{array}{l}\text { Salarios } \\
\text { medios }\end{array}$ & $\begin{array}{l}\text { salarios } \\
\text { altos }\end{array}$ & $\begin{array}{l}\text { Salarios } \\
\text { muy } \\
\text { altos }\end{array}$ \\
\hline $\begin{array}{l}\text { Primario } \\
\text { Independiente } \\
\text { Superior } \\
\end{array}$ & $0,20 \%$ & $19,17 \%$ & $54,15 \%$ & $4,94 \%$ & $21,54 \%$ & $1,71 \%$ & $26,45 \%$ & $63,74 \%$ & $8,10 \%$ \\
\hline $\begin{array}{l}\text { Primario } \\
\text { Independiente } \\
\text { Inferior }\end{array}$ & $4,62 \%$ & $49,64 \%$ & $31,02 \%$ & $0,58 \%$ & $14,14 \%$ & $0,32 \%$ & $42,95 \%$ & $49,52 \%$ & $7,21 \%$ \\
\hline $\begin{array}{l}\text { Primario } \\
\text { Dependiente } \\
\text { de Servicios }\end{array}$ & $3,20 \%$ & $68,68 \%$ & $10,14 \%$ & $0,71 \%$ & $17,26 \%$ & $17,12 \%$ & $60,18 \%$ & $21,98 \%$ & $0,72 \%$ \\
\hline $\begin{array}{l}\text { Primario } \\
\text { Dependiente } \\
\text { Industrial } \\
\end{array}$ & $5,66 \%$ & $64,37 \%$ & $5,66 \%$ & $0,00 \%$ & $24,32 \%$ & $18,22 \%$ & $78,36 \%$ & $3,42 \%$ & $0,00 \%$ \\
\hline $\begin{array}{l}\text { Secundario de } \\
\text { transición }\end{array}$ & $27,68 \%$ & $58,42 \%$ & $3,28 \%$ & $0,00 \%$ & $10,61 \%$ & $46,37 \%$ & $51,10 \%$ & $2,31 \%$ & $0,23 \%$ \\
\hline $\begin{array}{l}\text { Secundario } \\
\text { débil }\end{array}$ & $57,65 \%$ & $24,87 \%$ & $0,10 \%$ & $0,00 \%$ & $17,38 \%$ & $74,30 \%$ & $24,98 \%$ & $0,72 \%$ & $0,00 \%$ \\
\hline
\end{tabular}

Fuente: elaboración propia a partir de los datos de la ECVT 1999 y 2008

Tabla 6. Distribución de los trabajadores de acuerdo con el género según conglomerado.

\begin{tabular}{|l|r|r|r|r|}
\hline & \multicolumn{1}{|l|}{1999} & \multicolumn{1}{l|}{2008} \\
\hline & \multicolumn{1}{|l|}{ Hombre } & \multicolumn{1}{l|}{ Mujer } & \multicolumn{1}{l|}{ Hombre } & \multicolumn{1}{c|}{ Mujer } \\
\hline Primario Independiente Superior & $85,77 \%$ & $14,23 \%$ & $39,91 \%$ & $60,09 \%$ \\
\hline Primario Independiente Inferior & $48,48 \%$ & $51,52 \%$ & $87,56 \%$ & $12,44 \%$ \\
\hline Primario Dependiente de Servicios & $70,46 \%$ & $29,54 \%$ & $41,17 \%$ & $58,83 \%$ \\
\hline Primario Dependiente Industrial & $95,59 \%$ & $4,41 \%$ & $99,91 \%$ & $0,09 \%$ \\
\hline Secundario de transición & $86,21 \%$ & $13,79 \%$ & $47,98 \%$ & $52,02 \%$ \\
\hline Secundario débil & $19,98 \%$ & $80,02 \%$ & $25,50 \%$ & $74,50 \%$ \\
\hline
\end{tabular}

Fuente: elaboración propia a partir de los datos de la ECVT 1999 y 2008 
Tabla 7. Distribución de los trabajadores de acuerdo con sui país de nacimiento según conglomerado.

\begin{tabular}{|l|r|r|r|r|}
\hline & \multicolumn{1}{|l|}{1999} & \multicolumn{1}{l|}{$\mathbf{l}$} \\
\hline & \multicolumn{1}{|l|}{ España } & \multicolumn{1}{l|}{ extranjero } & \multicolumn{1}{l|}{ España } & extranjero \\
\hline Primario Independiente Superior & $94,27 \%$ & $5,73 \%$ & $98,06 \%$ & $1,94 \%$ \\
\hline Primario Independiente Inferior & $98,41 \%$ & $1,59 \%$ & $96,20 \%$ & $3,80 \%$ \\
\hline Primario Dependiente de Servicios & $99,47 \%$ & $0,53 \%$ & $98,92 \%$ & $1,08 \%$ \\
\hline Primario Dependiente Industrial & $98,98 \%$ & $1,02 \%$ & $87,25 \%$ & $12,75 \%$ \\
\hline Secundario de transición & $94,64 \%$ & $5,36 \%$ & $85,01 \%$ & $14,99 \%$ \\
\hline Secundario débil & $95,01 \%$ & $4,99 \%$ & $79,90 \%$ & $20,10 \%$ \\
\hline
\end{tabular}

Fuente: elaboración propia a partir de los datos de la ECVT 1999 y 2008

Tabla 8. Distribución de los trabajadores de acuerdo con la satisfacción general que expresan según conglomerado.

\begin{tabular}{|l|r|r|r|r|r|r|}
\hline \multicolumn{7}{|l|}{1999} \\
\end{tabular}

Fuente: elaboración propia a partir de los datos de la ECVT 1999 y 2008 
Tabla 9. Distribución de los trabajadores de acuerdo con si están o no afiliados a un sindicato según conglomerado.

\begin{tabular}{|l|r|r|r|r|r|}
\hline \multicolumn{1}{|l|}{1999} & \multicolumn{1}{l|}{$\mathbf{2 0 0 8}$} \\
\hline & \multicolumn{1}{l|}{ sí } & \multicolumn{1}{l|}{ no } & \multicolumn{1}{l|}{ NS/NC } & \multicolumn{1}{l|}{ sí } & \multicolumn{1}{l|}{ no } \\
\hline Primario Independiente Superior & $13,24 \%$ & $85,57 \%$ & $1,19 \%$ & $30,22 \%$ & $69,78 \%$ \\
\hline Primario Independiente Inferior & $23,52 \%$ & $73,88 \%$ & $2,60 \%$ & $25,52 \%$ & $74,48 \%$ \\
\hline Primario Dependiente de Servicios & $46,44 \%$ & $49,64 \%$ & $3,91 \%$ & $43,24 \%$ & $56,76 \%$ \\
\hline Primario Dependiente Industrial & $7,92 \%$ & $90,72 \%$ & $1,36 \%$ & $17,62 \%$ & $82,38 \%$ \\
\hline Secundario de transición & $14,11 \%$ & $84,57 \%$ & $1,31 \%$ & $5,07 \%$ & $94,93 \%$ \\
\hline Secundario débil & $3,02 \%$ & $96,57 \%$ & $0,42 \%$ & $10,61 \%$ & $89,39 \%$ \\
\hline
\end{tabular}

Fuente: elaboración propia a partir de los datos de la ECVT 1999 y 2008

\subsection{Análisis CHAID}

En el análisis CHAID lo que se pretende es observar para las tres variables que han sido señaladas como dependientes (sector productivo, tipo de contrato y salario) cuáles son las variables intervinientes que en mayor medida determinan su comportamiento. Esto permitirá considerar cuáles son les elementos más importantes que van a producir la segmentación de los mercados de trabajo, al menos en las variables concretas que se consideran, y pueden dar importantes pistas sobre la lógica de la aparición o consolidación de una nueva clase trabajadora en los servicios.

\subsubsection{Sector productivo}

Se detecta de forma general para 1999 una distribución en la que los hombres se encuentran ocupados de forma más intensa en actividades de resto del sistema productivo y donde las mujeres se encuentran ocupadas en los servicios, por lo que podríamos hablar de una feminización de las actividades terciarias. En todo caso es importante hacer ver que los hombres con mayores formaciones también se ocupan en los servicios y que, al mismo tiempo, son las mujeres las que monopolizan la presencia en actividades de servicios manuales. Es por esto que se habla de segmentación por género del mercado de trabajo. De la misma manera, y teniendo en cuenta que el proceso de terciarización no se ha detenido entre 1999 y 2008, creo que podría apuntarse una especie de polarización en las oportunidades de empleo en los servicios, que se sitúan o bien en la parte alta o bien en la parte baja del mercado de trabajo. En realidad esto no es extraño si tenemos en cuenta que la mayor parte de las ocupaciones, excepto si consideramos la construcción en el período señalado, están creándose en los sectores de servicios: de esta manera es lógico que aparezcan en los servicios las buenas y las malas oportunidades de trabajo. En definitiva, y para 2008, las variables que influyen respecto la distribución de los trabajadores por sectores son muy semejantes a las que tenían un peso decisivo en 1999.

Es muy significativo que para el ámbito privado el género sea la variable que más importancia explicativa tiene tanto en 1999 como en 2008, ya que este hecho 
remite de nuevo a la idea de la segmentación por género de los mercados de trabajo: si tenemos en cuenta que muchos de los ocupados en los servicios manuales van a tener ocupaciones de peor calidad que la media, el mayor peso de las mujeres en estos sectores productivos es una fuente de discriminación por género dentro los mercados de trabajo que no parece desaparecer a lo largo de los años. En todo caso es necesario apuntar el descenso en números relativos de aquellos que se ocupan en estos servicios manuales. Considerar esto junto al crecimiento de las actividades terciarias en general nos lleva a valorar la posibilidad de que la polarización en las actividades de servicios -o de mucha calidad o de muy poca calidad- se haya estado resolviendo en el período estudiado a favor de ocupaciones con mejores condiciones de trabajo. Sea como sea no hay que olvidar que el sector que hemos denominado como resto de servicios es suficientemente amplio como para incluir una gran cantidad de subsectores productivos en los que se puede estar dando mucha diversidad en cuanto a las condiciones de trabajo.

Por último, el peso de la formación tanto en 1999 como, sobre todo, en 2008 es decisivo en muchas ocasiones a la hora de considerar en qué sector productivo van a estar ocupados los trabajadores. Para el caso de los hombres en el ámbito privado, y esto sucede tanto para 1999 como para 2008, bajas formaciones implican que habrá mayor ocupación en actividades de resto del sistema productivo, básicamente industria y construcción. En el caso de las mujeres, para 1999 la variable determinante era la tamaño de la empresa, pero en 2008 es la formación y así puede comprobarse que bajas formaciones -en especial por lo que respecta a las que tienen formación primaria y secundaria- hacen que las mujeres estén ocupadas en las actividades de servicios manuales, que son las que a priori tienen peores condiciones de trabajo. De esta manera toma fuerza el argumento de que los hombres con bajas formaciones no parecen condenados a ocupar las peores condiciones en el mercado de trabajo, por lo menos en la misma medida que las mujeres.

\subsubsection{Tipo de contrato}

Para 1999 la temporalidad se distribuye según la edad, como era de esperar, pero también en muchos casos según el género, hecho que insiste en la discriminación por género en los mercados de trabajo. En edades superiores, por encima de los 45 años, creo que es muy significativo valorar la tasa de temporalidad de aquellos ocupados en empresas pequeñas en los servicios manuales y en el resto del sistema productivo, ya que se acerca casi al $25 \%$ de los efectivos y, por tanto, este es también una dato importando por lo que respecta a la consideración de determinados colectivos de trabajadores que, a pesar de disponer de características que hipotéticamente los alejarían de la precariedad, se encuentran aún atrapados en ella: en este caso hablaríamos de transiciones anormales desde la formación hasta el empleo o desde la juventud hasta las atribuciones que se les suponen a los trabajadores de edad adulta. Para el año 2008 los datos indican que hay una mayor heterogeneidad que en 1999 a la hora de considerar las posibilidades de los 
trabajadores de disponer o no de un contrato temporal. A la edad, a la formación y al género se une en este caso como factor bastante importante el país de procedencia ya que en 1999 el número de trabajadores extranjeros aún no era muy numeroso; esto viene a completar el panorama que sitúa como grupos más desfavorecidos en el mercado de trabajo a las mujeres, a los jóvenes y a los trabajadores inmigrantes, que están ocupados en los segmentos más precarios del mercado de trabajo y que sufren las condiciones más complicadas.

\subsubsection{Salarios}

Para 1999 el peso del género y la edad compensa y matiza la esperada influencia de la formación en la distribución de los salarios. Los mayores salarios para las edades superiores son también un efecto esperado de la antigüedad y la experiencia en las ocupaciones, pero el peso del género muestra la existencia de un cierto grado de discriminación en los mercados de trabajo en contra de las trabajadoras que, además, en mayor o menor medida se produce a todas las categorías de formación. Para el caso de 2008 esto no solo no desaparece sino que parece consolidarse, junto a otras fuentes de discriminación que profundizan la segmentación de los mercados a partir de los colectivos de trabajadores. En definitiva, para 2008, se repiten los mismos elementos que en 1999 respeto la construcción de las categorías salariales, insistiendo y haciendo más fuerte la tendencia a segmentar por género el mercado de trabajo, lo que es sintomático de un mercado de trabajo que genera distintas oportunidades según las características de los trabajadores. De forma general los datos insisten en la segmentación de los mercados de trabajo por género y edad, viéndose en alguno de los casos que la segmentación también actúa por país de procedencia. Hay que tener en cuenta, sin embargo, que son las categorías de formación las que actúan como principal elemento clasificador y, para el caso de los universitarios, es importante hacer ver que la edad pesa más que el género en la distribución de los salarios, de forma que en 2008 aquellas bien formadas tienen más probabilidades de tener menos diferencias salariales respecto trabajadores masculinos con la misma formación. Sea como sea, incluso entre estos trabajadores, finalmente aparece el género como ente diferenciador.

\section{Conclusiones}

Las nuevas condiciones económicas y las nuevas estructuras productivas han provocado un cambio en la forma como se producen los bienes y servicios así como en la naturaleza de estos mismos bienes y servicios. También sabemos como todas estas transformaciones tienen consecuencias en la fuerza de trabajo que desarrolla las ocupaciones, de manera que si hay nuevas ocupaciones y tareas o las proporciones entre sectores y subsectores productivos han variado, es lógico que en el mismo sentido haya variado la fuerza de trabajo que las lleva a cabo, tanto cuantitativa como cualitativamente. Así, las transformaciones que se han hecho 
efectivas para la fuerza de trabajo hacen que ésta tome formas diferentes a la tradicional clase obrera, de manera que podemos considerar que, en una interpretación más extrema, habría una nueva clase trabajadora que convive junto a la clase obrera industrial o que, por lo menos en una interpretación más conservadora, esta clase trabajadora ahora es mucho más heterogénea e incluye elementos que desarrollan su trabajo en la industria pero también, y mayoritariamente, en los servicios. En este sentido, las actividades de servicios toman el protagonismo en cuanto a número de asalariados y eso podría significar que sus trabajadores pasan a ser considerados como la más característica clase trabajadora en substitución de la antigua clase obrera, de manera que hay una evidente mutación en esta clase que afectará al conjunto de la estructura social de los países desarrollados.

En los años posteriores a la segunda guerra mundial la preocupación por averiguar la estructura de clases de las sociedades desarrolladas ocupó muchos esfuerzos a los científicos sociales. La existencia de las clases medias (Wright, 1983) (Lockwood, 1962), la forma como adscribir a los distintos individuos a unas u otras clases (Wright, 1979 y 1994) y la naturaleza familiar de ésta (Humphries, 1982) (Pahl, 1991) o como nombrar a estas clases sociales para que no refirieran de forma directa a aspectos políticos sino únicamente sociales y, por tanto, facilitaran la adscripción de los individuos a unas u otras sin que esto supusiera ningún sesgo de tipo ideológico, fueron importantes temas y elementos de debate y búsqueda.

Siguiendo el trabajo de Wright (Wright, 1994) la forma más correcta para establecer la estructura de clases de una sociedad debe ver con lo que él llama concepto de estructura de clases centrado en la explotación. Esta construcción teórica es más compleja que las otras alternativas que considera pero el autor defiende que tiene importantes posibilidades explicativas. De acuerdo a Wright hay tres dimensiones en cuanto a las relaciones de explotación a lo largo de las que un individuo puede situarse. La primera de las dimensiones (bienes en medios de producción) vendría a considerar la posesión o no de los medios de producción; la segunda (bienes en la organización) la posesión o no de control efectivo sobre la coordinación e integración de la división del trabajo; la tercera (bienes en credenciales) se refiere a las calificaciones académicas y profesionales de las que una persona dispone. Así, consideramos que por lo que respecta a la composición de las clases sociales la posición en el mercado de trabajo es determinante.

Una vez establecido esto, el análisis de clases debe enfrentarse a las colectividades de trabajadores asalariados que, aunque participan en el mercado de trabajo en actividades de servicios ( o precisamente por ello), desarrollan su actividad en condiciones de trabajo complicadas en cuanto al salario a que reciben, a la inestabilidad que pueden sufrir, a la escasa presencia de tecnología y de necesidades de formación, etc. de manera que puede concluirse que determinadas ocupaciones de servicios -incluso en subsectores prestigiados- no se adecuan a aquello definido para las clases medias, por lo menos en cuanto a condiciones de empleo se refiere y especialmente si consideramos las ocupaciones y subsectores de servicios donde es necesaria una presencia intensiva del trabajador: hostelería, 
comercio al por menor y en grandes superficies, determinados servicios a las empresas como la limpieza, servicios personales, etc. Son trabajadores que no poseen los medios de producción, que no controlan los ritmos ni la organización de su trabajo y a los que su formación (existente o no, reglada o informal) no les aporta ningún tipo de ventaja en el desarrollo concreto de su tarea: las condiciones para la definición de clase trabajadora según Wright.

Sabemos que el crecimiento de todos estos sectores genera una importante masa de trabajadores que no están considerados dentro de la clase obrera tradicional de cariz industrial, pero también sabemos que los orígenes sociales de estos trabajadores, los colectivos de los que proceden, sus expectativas y sus necesidades, son diferentes, y todo esto es fruto de los procesos de fragmentación social, consecuencia de la segmentación de los mercados de trabajo (Tezanos, 2001). En todo caso es posible que las nuevas clases trabajadoras, que ahora incluyen a muchos trabajadores en servicios y que tienen una gran presencia de mujeres e inmigrantes, tienen también una base objetiva y real de condiciones de trabajo y de vida que, fuera de las identificaciones entre los trabajadores que pueden tener lugar, supone por tanto su existencia como colectividad no solo en el mundo del trabajo, en los segmentos secundarios -principalmente a los subsectores de servicios-, sino también a nivel social.

Esta hipótesis se refiere a la existencia concreta de lo que he denominado nueva clase trabajadora de servicios, y que podría venir definida por los perfiles débiles o secundarios que se han descrito. Los datos resultantes podrían acabar confirmando la existencia de esta colectividad de trabajadores. En todo caso es necesario señalar que el peso de los segmentos secundarios entre 1999 y 2008 ha disminuido en aproximadamente 5 puntos porcentuales, hecho que vendría a invalidar la parte de la hipótesis que habla del incremento de la importancia de este colectivo. Frente a esto es necesario argumentar en primer lugar que el sexto clúster, el segmento secundario débil, crece a lo largo del período cuando la tendencia establecida por el cuarto y quinto clústeres apuntaba hacia un descenso: esto en un momento de expansión económica ya es significativo por sí mismo; y hay que recordar igualmente que este segmento secundario débil es el clúster más numeroso tanto en 1999 como en 2008.

Además, y tal y como se ha señalado a lo largo del texto, el quinto clúster de 1999 tiene características de empleo que le sitúan próximo al segmento primario dependiente industrial; sin embargo el quinto clúster (segmento secundario de transición) de 2008 tiene características mucho más semejantes a las del segmento secundario débil y es un segmento secundario de servicios en la línea del descrito para las nuevas clases trabajadoras de servicios. En este sentido la aparición de dos clústeres precarios de servicios, que agrupan a una gran cantidad de colectivos y situaciones diferentes es un elemento de subsegmentación de la precariedad -dentro de las condiciones precarias a los servicios manuales, las del segmento secundario débil empeoran las del segmento secundario de transición- así como de consolidación de la tendencia que en 1999 ya situaba a los sectores de servicios manuales en la mayor precariedad. 
Las evidencias muestran la existencia de este sector de características laborales precarias que se sitúa básicamente en los servicios. Creo, además, que hay varias características de cariz social que hay que señalar -abundancia de mujeres, inmigrantes, trabajadores de edades más jóvenes, cierta presencia de trabajadores con una formación elevada- y que nos pueden llegar a dar una imagen global del grupo. Pero hay también otros elementos que cambian entre 1999 y 2008 y que me parece necesario comentar ya que van a señalar como esta subclase trabajadora también ha venido complejizándose en los últimos años, de igual manera que el mercado de trabajo tomado de manera general.

En este sentido los trabajadores que caen en la precariedad también han pasado a ser de empresas de mayor tamaño y, de igual manera, también trabajadores de sectores económicos antes ajenos a los perfiles débiles ahora participan de ella. El motivo de esto hay que buscarlo en la posición dentro de la estructura productiva de muchas de estas actividades -es decir, en su naturaleza subalterna- pero también en el poco valor añadido que estas actividades otorgan a su producto final: son actividades basadas en la intensidad de la mano de obra. Con esta estrategia empresarial es muy complicado que las condiciones de trabajo mejoren para los empleados en estos subsectores, ya que un aumento de las prestaciones a los ocupados -si no viene acompañado de una mejora en el producto o servicio- implica que la actividad pierde su rentabilidad en el mercado. Y en definitiva los datos de los análisis nos señalan, así, que esta forma de competir, en un momento de expansión económica, lejos de remitir se ha venido extendiendo.

Es interesante hacer ver la existencia de estos segmentos de características laborales precarias a partir de la consistencia de los distintos análisis CHAID a la hora de mostrar cuáles son los grupos que se quedan con las peores condiciones de trabajo. En este sentido los que sufrirían la precariedad serían las mujeres, los más jóvenes, los trabajadores inmigrantes, aquellos con formaciones más bajas, los que trabajan en empresas de tamaños reducidos y en determinados subsectores económicos -que son aquellos que hemos renombrado de servicios manuales-, los que presentan más bajos salarios, los que alargan más sus horarios o sufren más las jornadas parciales, los que disponen de contratos temporales, tienen más bajos grados de satisfacción con su trabajo $\mathrm{y}$, en definitiva, aquellos que tienen peores condiciones de trabajo. Que estos sean los colectivos que coinciden de una manera bastante fiel con los que conforman los segmentos secundarios de acuerdo al análisis clúster da a los resultados una importante solidez.

En definitiva, uno de los elementos relacionados con la hipótesis y que creo que se puede observar a partir del análisis CHAID -pero que también ha estado apuntado a partir del análisis clúster- es que los segmentos secundarios presentan, dentro de ellos, una tendencia a incorporar trabajadores de realidades cada vez más diversas. Es decir, aparte del incremento de la heterogeneización dentro del mercado de trabajo en general y de la que es muestra la construcción de una tipología de seis segmentos a la hora de considerar este mercado, hay que señalar que dentro de los propios segmentos y, en especial, dentro los segmentos secundarios, la heterogeneidad también ha sido creciente. En este punto creo que 
esto puede comprobarse a partir de la complejización de las relaciones entre las variables así como partir del aumento, entre 1999 y 2008, del número de variables intervinientes en cada uno de los análisis que se han planteado.

\section{Bibliografía}

Alós, R. (2007). Mercat, Classe i Persona en les Relacions Laborals. Entre la Individualitat i l'Acció Col-lectiva. Tesis Doctoral. Mayo de 2007. Bellaterra. Universitat Autònoma de Barcelona.

Alós, R.; Jódar, P. (2005). Relaciones laborales segmentadas en mercados de trabajo segmentados en Gaceta Sindical, Núm. 5, 224-249.

Amable, M.; Benach, J. (2007). Desigualdades en Salud Laboral en V. Navarro (Coord.) La situación Social en España II (pp. 257-292), Madrid: Biblioteca Nueva.

Amable, M.; Benach, J. y González, S. (2001). La precariedad laboral y su repercusión sobre la salud: conceptos y resultados preliminares de un estudio multimétodos en Archivo de Prevención de Riesgos Laborales. Núm. 4, Vol. 4, 169-184.

Baglioni, G. (1992). Las relaciones laborales en Europa en el decenio 1980-1990 en Guido. Baglioni i Colin Crouch (eds) Las Relaciones Laborales en Europa. El desafio de la flexibilidad (pp. 21-67), Madrid: Ministerio de Trabajo y Seguridad Social.

Banyuls, J.; Cano, E. y Aguado, E. (2011). La incidencia del salario mínimo interprofesional en sectores de bajos salarios en Cuadernos de Relaciones Laborales. Vol. 29, Núm. 2, 363-389.

Blanch, J. M. (2003). Trabajar en la modernidad industrial en Josep M. Blanch (Coord.) Teoría de las Relaciones Laborales. Fundamentos (pp. 19-148), Barcelona: UOC.

Braverman, H. (1974). Labor and Monopoly Capital. The Degradation of Work in the Twentieth Century. New York: Monthly Review Press.

Burawoy, M. (1979). Manufacturing Consent. Changes in the labor process under monopoly capitalism. Chicago: The University of Chicago Press.

Cano, E. (1997). Canvi Socioeconòmic i Precarització Laboral. Tesis Doctoral. València. Universitat de València. 
Cano, E. (1998). La lógica de la precariedad laboral: el caso de la industria valenciana del mueble en Cuadernos de Relaciones Laborales, Núm. 13, 207-227.

Cano, E. (2004). "Formas, percepciones y consecuencias de la precariedad" en Mientras Tanto. Núm. 93, 67-81.

Cano, E. (2007). La extensión de la precariedad laboral como norma social en Sociedad y Utopía. Revista de Ciencias Sociales. Núm. 29, 117-137.

Cardenal, M. E. (2006). La universidad como dispositivo de colocación social. Movilidad y reproducción en la era de la precariedad laboral en Revista de Educación. Núm. 341, 281-299.

Dickens, W.; Lang, K. (1992). Labor Market Segmentation Theory: reconsidering the evidence en NBER (National Bureau of Economic Research) Working Papers Series. Núm. 4087, 82 pp.

Doeringer, Peter.; Piore, M. (1985). Mercados internos de trabajo y análisis laboral. Madrid: Ministerio de Trabajo y Seguridad Social.

Gordon, D.; Edwards, R. y Reich, M. (1986). Trabajo Segmentado, Trabajadores Divididos. La transformación histórica del trabajo en los Estados Unidos. Madrid: Ministerio de Trabajo y Seguridad Social.

Humphries, J. (1982). Class Struggle and the Persistence of the Working-Class Family en Anthony Gigdden y David Held (eds.) Classes, Power and Conflict (pp. 470-490). Los Angeles: University of California Press.

Iglesias, C.; Llorente, R. (2006). ¿Integración o segmentación laboral de los inmigrantes en el mercado de trabajo? Un análisis de cohortes en Documentos de Trabajo del Instituto Universitario de Análisis Económico y Social. Núm. 07/2006. Alcalá de Henares: Universidad de Alcalá.

Kern, Horst; Schumann, M. (1988). El Fin de la División del Trabajo. Racionalización en la Producción Industrial. Madrid: Ministerio de Trabajo y Seguridad Social.

Lockwood, D. (1962). El trabajador de clase media. Madrid: Aguilar.

López Peláez, A. (2005). Excluidos pero trabajadores: el círculo vicioso de los trabajadores con bajo salario en España. En Cuadernos de Relaciones Laborales. Vol. 23. Núm. 1, 153-174. 
López Roldán, P. (1996). La construcción de una tipología de segmentación del mercado de trabajo en Papers, Revista de Sociología. Núm. 48, 41-58.

Marshall, A. (1992). Secuelas del paro: el nuevo papel del trabajo temporal y del trabajo a tiempo parcial en Europa Occidental en Gerry Rodgers y Janine Rodgers (comp) El Trabajo Precario en la Regulación del Mercado Laboral. Crecimiento del Empleo Atípico en Europa Occidental. (pp.43-90) Madrid: Ministerio de Trabajo y Seguridad Social.

Medialdea, B.; Álvarez, N. (2005). Ajuste neoliberal y pobreza salarial: los working poor en la Unión Europea, en Viento Sur. Núm. 82, 56-64.

Miguélez, F. (2004). La flexibilidad laboral en Trabajo. Revista Andaluza de relaciones Laborales. Núm. 1, 17-36.

Miguélez, F. (2005). Trabajo Flexible, Empleo Flexible. Manuscrit, 23 pàgines. QUIT, UAB. Ponència presentada en el Seminari Europeu El modelo social europeo. Una visión alternativa Diciembre 2005. Consultado el 10 de octubre de 2013 en: http://www.ucm.es/data/cont/docs/183-2013-05-08Ponencia_Fausto\%20Miguelez.pdf

OIT (Organización Internacional del Trabajo) (1998). Resolución concerniente a la medición del subempleo y las situaciones de empleo inadecuado, adoptada por la decimosexta Conferencia Internacional de Estadísticos del Trabajo. Octubre de 1998. Consultado el 10 de octubre de 2013 en: http://www.ilo.org/wcmsp5/groups/public/---dgreports/--stat/documents/normativeinstrument/wcms_087489.pdf

Pahl, R. (1991). Divisiones del Trabajo. Madrid: Ministerio de Trabajo y Seguridad Social.

Paoli, P. (1999). Condiciones de trabajo. Los nuevos retos para las políticas de salud en el trabajo en Cuadernos de Relaciones Laborales. Núm. 14, 173-180.

Pinilla, F. J. (2004). Intensificación del esfuerzo de trabajo en España en Cuadernos de Relaciones Laborales, Vol. 22, Núm. 2, 117-135.

Pollert, A. (1991). Farewell to Flexibility? Londres: Basil Blackwell.

Prieto, C. (Coord.); Arnal, M.; Caprile, M. y Potrony, J. (2009). La calidad del empleo en España. Una aproximación teórica y empírica. Madrid: Ministerio de Trabajo e Inmigración. 
Recio, A. (2007). Precariedad laboral: del neoliberalismo a la búsqueda de un modelo alternativo. Comunicació a congrés El desafio del desarrollo humano. Propuestas locales para otra globalización. Organitzat per HEGOA, Instituto de Estudios sobre Desarrollo y Cooperación Internacional. Bilbao, 8, 9 i 10 de febrer de 2007. Consultado el 10 de octubre de 2013 en: http://www.hegoa.ehu.es/congreso/bilbo/komu/1_Derechos/2_Albert-Recio.pdf

Recio, A. (2011). Segmentación laboral y acción sindical en Gaceta Sindical: Reflexión y Debate. Núm. 16, 163-176.

Rubery, J. (1978). Structured labour markets, worker organisation and low pay" en Cambridge Journal of Economics. Vol. 2, 17-36.

Rubery, J. Wilkinson, F. (1981). Outwork and Segmented Labour Markets en F. Wilkinson (ed.) The Dynamics of Labour Market Segmentation (pp.115-132) Londres: Academic Press.

Tezanos, J. F. (2001). La sociedad dividida. Estructuras de clases y desigualdades en las sociedades tecnológicas. Madrid: Biblioteca Nueva.

Wright, E. O. (1979). Class structure and income determination. Londres: Academic Press.

Wright, E. O. (1983). Clase, crisis y estado. Madrid: Siglo XXI.

Wright, E. O. (1994). Clases. Madrid: Siglo XXI. 